\title{
VIRMS: A VEHICLE INFORMATION AND ROAD MONITORING SYSTEM
}

\section{Fabio Arnéz and Alex Villazón}

\begin{abstract}
Intelligent Transport Systems (ITS) are emerging technologies for building collaborative vehicular networks to increase road safety and to improve driver's experience. Unfortunately these technologies require heavy infrastructure to be deployed inside and outside the vehicle that is difficult to extend. In this article we present VIRMS (Vehicle Information and Road Monitoring System), an ITS that is based on low-cost and small footprint client and server infrastructure that was designed to increase vehicular security and reduce accident rates along highways. The VIRMS remote client device is an on board vehicle electronic device that gathers data from sensors and processes the collected data that is sent to the VIRMS server in order to keep drivers informed with precise context information through the detection and identification of events (accidents, traffic jams, bad weather conditions, etc.) along the roads. A prototype running tests on Bolivian highways show that VIRMS can give a technological answer to a real problem where road safety is one of the highest issues and cause of mortality.
\end{abstract}

Keywords: Intelligent Transportation System, Embedded System, Electronic Vehicle Device, Real-Time Operating System, Communication Protocol. 